\title{
NÁUSEAS, VÔMITOS E QUALIDADE DE VIDA DE MULHERES COM CÂNCER DE MAMA EM TRATAMENTO QUIMIOTERÁPICO
}

\author{
Thais de Oliveira GOZZO ${ }^{a}$, Aline Maria Bonini MOYSÉS ${ }^{b}$, \\ Pamina Roberta da SILVA' ${ }^{\mathrm{c}}$, Ana Maria de ALMEIDA ${ }^{\mathrm{d}}$
}

\section{RESUMO}

Objetivou-se avaliar a qualidade de vida $(\mathrm{QV})$ de mulheres com câncer de mama em tratamento quimioterápico e identificar a ocorrência de náuseas e vômitos durante o tratamento. Os dados foram coletados com a aplicação do instrumento da Organização Europeia de Pesquisa e Tratamento de Câncer, EORTC-QLQ-C30, na versão em português, bem como do módulo para câncer de mama BR-23, aplicados antes, no meio e ao final do tratamento. Das 79 mulheres incluídas, 93\% apresentaram náuseas e $87 \%$ vômitos pelo menos uma vez durante o tratamento. A QV apresentou pequena diminuição durante o tratamento. O coeficiente alfa de Cronbach para cada aplicação dos questionários foi de 0,890492, 0,936392 e de 0,937639. A disponibilidade de informações sobre o tratamento e de orientações quanto ao manejo da náusea e do vômito é crucial para o gerenciamento adequado das toxicidades da quimioterapia.

Descritores: Neoplasias da mama. Náusea. Vômito. Qualidade de vida. Quimioterapia.

\section{RESUMEN}

Evaluar la calidad de vida (QOL) de las mujeres con cáncer de mama durante la quimioterapia e identificar el acontecimiento de náuseas y vómitos durante el tratamiento. Se recogieron datos con la aplicación del instrumento de la Organización Europea para la Investigación y Tratamiento del Cáncer, EORTC-QLQ-C3O versión en portugués y módulo para el cáncer de mama BR-23 aplicado antes, en la mitad y al final del tratamiento. Se incluyeron 79 mujeres, el 93\% tuvo náuseas, el 87\% vómitos al menos una vez durante el tratamiento. La QOL presentó una ligera disminución durante el tratamiento. El coeficiente alfa de Cronbach para cada aplicación de los cuestionarios fue 0.890492, 0.936392 y 0.937639. La disponibilidad de informaciones sobre el tratamiento y directrices sobre el manejo de la náusea y vómito es fundamental para la correcta gestión de las toxicidades de la quimioterapia.

Descriptores: Neoplasias de la mama. Náusea. Vómito. Calidad de vida. Quimioterapia.

Título: Náuseas, vómitos y calidad de vida en mujeres con cáncer de mama en la quimioterapia.

\section{ABSTRACT}

The aim of this study was to assess the quality of life (QoL) of women with breast cancer during chemotherapy and to identify the incidence of nausea and vomiting during the treatment. Data were assessed with the application of the instrument of the European Organization for Research and Treatment of Cancer, EORTC-QLQ-C3O Portuguese version and breast cancer module BR-23, which was applied before, in the middle and in the end of the treatment. The participants were 79 women, of which 93\% had nausea and 87\% had vomited at least once during the treatment. QoL showed a slight decrease during treatment. Cronbach's alpha for each application of the questionnaires was $0.890492,0.936392$ and 0.937639 . The availability of treatment information and guidelines on the management of nausea and vomiting is crucial for the proper management of the toxicities of chemotherapy.

Descriptors: Breast neoplasm. Nausea. Vomiting. Quality of life. Drug therapy.

Title: Nausea, vomiting and quality of life in women with breast cancer receiving chemotherapy.

a Enfermeira. Professora Doutora do Departamento Materno Infantil e Saúde Pública da Escola de Enfermagem de Ribeirão Preto da Universidade de São Paulo. Ribeirão Preto- São Paulo, Brasil.

b Enfermeira. Mestranda do Programa de Pós-Graduação Enfermagem em Saúde Pública da Escola de Enfermagem de Ribeirão Preto da Universidade de São Paulo. Ribeirão Preto- São Paulo, Brasil.

c Enfermeira. Mestranda do Programa de Pós-Graduação Enfermagem em Saúde Pública da Escola de Enfermagem de Ribeirão Preto da Universidade de São Paulo. Ribeirão Preto- São Paulo, Brasil.

d Enfermeira. Professora Associada do Departamento de Enfermagem Materno-Infantil e Saúde Pública da Escola de Enfermagem de Ribeirão Preto da Universidade de São Paulo. Ribeirão Preto- São Paulo, Brasil. 


\section{INTRODUÇÃO}

Os eventos adversos (EAs) causados pelos quimioterápicos relacionam-se ao fato da sua inespecificidade pelas células tumorais e os efeitos citotóxicos nas células normais. Esses eventos predominam nas células que estão em constante divisão celular, como as do tecido hematopoiético, do tecido germinativo, do folículo piloso e do revestimento gastrointestinal ${ }^{(1)}$. Apesar de aumentar a sobrevida, a quimioterapia, bem como as terapias hormonais influenciam negativamente na qualidade de vida $(\mathrm{QV})$ devido a estes $\mathrm{EAs}^{(2)}$.

Dentre os EAs gastrointestinais podemos citar náuseas, vômitos, mucosite, diarréia, anorexia, estomatite, desconforto ou dor abdominal, porém os referidos pelos pacientes oncológicos como os mais estressantes e incômodos, são a náusea e o vômito ${ }^{(3-4)}$. Ocorrem juntos ou isolados, entretanto devem ser avaliados separadamente, pois são eventos distintos, com causas também distintas ${ }^{(5)}$.

A incidência de náuseas e vômitos está relacionada primariamente com o potencial emético das drogas que varia desde muito alto, quando provocam vômitos em mais de $90 \%$ dos pacientes, até potencialmente baixo, que ocasionam menos de $10 \%{ }^{(6)}$. Também está associada a variações como sexo, idade, ansiedade, consumo de álcool, radioterapia no trato gastrintestinal e expectativa de desenvolvimento de náuseas e vômitos. Fatores relacionados ao quimioterápico que podem influenciar na ocorrência destes eventos são: a própria droga utilizada, a dose, a combinação de drogas, a via, a velocidade de administração e o número de ciclos recebidos ${ }^{(7-8)}$.

Todas as drogas quimioterápicas possuem potencial emetogênico, que varia de intensidade. O potencial das drogas mais utilizadas nos protocolos para tratamento do câncer de mama está descrito a seguir ${ }^{(6,13)}$ : alto (acima de 90\%): ciclofosfamida (acima de $1500 \mathrm{mg} / \mathrm{m} 2$ ) e cisplatina; moderado (em torno de 30 a $90 \%$ ): ciclofosfamida (abaixo de $1500 \mathrm{mg} / \mathrm{m} 2$ ), epirrubicina, doxorrubicina; baixo (de 10 a $30 \%$ ): 5-fluouracil, paclitaxel, docetaxel, metotrexate, doxorrubicina lipossomal, gencitabina, trastuzumab e mínimo (abaixo de 10\%): vinorelbine.

Aproximadamente $50 \%$ dos pacientes com câncer irão sentir náuseas e vômitos durante o tratamento quimioterápico; vômitos induzidos por quimioterapia podem ser prevenidos em cerca de 70 a $80 \%$ dos pacientes com o uso correto de antieméticos, porém, o controle das náuseas, é mais limitado ${ }^{(9)}$.
A incapacidade para atingir um nível desejado de controle desses eventos em todos os pacientes é multifatorial, como a incapacidade de controlar a náusea e compreender seu mecanismo fisiológico, o uso continuado de regimes de quimioterapia emetogênica, a subestimação do relato desses eventos pelos profissionais, a falta de conformidade entre as diretrizes de antieméticos e quimioterápicos prescritos e o desenvolvimento segmentando de controle de vômitos em detrimento da náusea ${ }^{(8)}$.

Náusea induzida pela quimioterapia tem alto impacto sobre o estado nutricional e atividades de vida diária, podendo prejudicar a QV. Por ser a náusea um sintoma-chave associado a outros sintomas, como o vômito, é imperativo que maior atenção seja dada ao seu controle tanto por meio de medidas farmacológicas e também, por medidas não farmacológicas, como intervenções nutricionais ${ }^{(10)}$.

Tendo-se em vista que o tratamento quimioterápico acarreta um grande impacto tanto de ordem física como emocional nestas mulheres e observando que a assistência a estes sintomas são pouco valorizados em mulheres com câncer de mama, além de a instituição não ter um protocolo de cuidados e de manejo adequado para os pacientes em quimioterapia, o presente estudo teve como objetivos: identificar a ocorrência de náuseas e vômitos provocada pelas drogas utilizadas nos protocolos de quimioterapia neoadjuvante e adjuvante referida pelas mulheres seguidas no Ambulatório de Mastologia do Hospital das Clínicas da Faculdade de Medicina de Ribeirão Preto da Universidade de São Paulo (HCFMRP-USP), e avaliar a QV dessas mulheres no início, no meio e ao final do tratamento quimioterápico.

\section{MÉTODOS}

O presente estudo é um recorte da pesquisa intitulada "Avaliação da Qualidade de Vida, Ansiedade, Depressão e Toxicidade ao Tratamento Quimioterápico em Mulheres com Câncer de Mama”, que além da qualidade de vida avaliou sinais e sintomas relacionados aos eventos adversos do tratamento quimioterápico. Estudo quantitativo e prospectivo aprovado pelo Comitê de Ética do HCFMRP-USP, protocolo 765/2009.

Foram recrutadas mulheres com câncer de mama que iniciaram tratamento quimioterápico no período de abril de 2009 a março de 2010 , seguindo 
os critérios de inclusão: idade acima de 19 anos, iniciando o tratamento quimioterápico pela primeira vez. Os critérios de exclusão foram: mulheres que não pudessem se expressar individualmente; que apresentassem doença metastática ou outro tipo de tumor maligno no diagnóstico; que já tivessem recebido tratamento quimioterápico por câncer de mama ou outro tipo de tumor maligno e no ciclo gravídico puerperal.

O instrumento de coleta de dados continha informações quanto aos dados sócio-demográficos, esquema quimioterápico proposto para neoadjuvância ou adjuvância e ocorrência de náuseas e/ou vômitos após cada ciclo. Estas informações foram obtidas pela pesquisadora por meio de entrevista e em revisão do prontuário das mulheres participantes do estudo. Para avaliar a ocorrência de náuseas e vômitos as perguntas eram feitas no dia da quimioterapia, porém as mulheres respondiam questões relacionadas ao ciclo anterior. Para a avaliação da QV foi aplicado o instrumento da Organização Européia de Pesquisa e Tratamento de Câncer, EORTC QLQ-C30 (versão 3.0) traduzido e validado para o português ${ }^{(11)}$, em três momentos diferentes, ou seja, antes de iniciarem o tratamento quimioterápico, na metade e ao término do mesmo.

O instrumento QLQ C-30 é um questionário que contém trinta itens divididos em três domínios: Estado de Saúde Global, com duas questões de avaliação; Escalas Funcionais, com quinze itens para avaliação do funcionamento físico, do desempenho de papéis, de funcionamentos emocional, cognitivo e social; Escalas de Sintomas que são compostas por treze itens de avaliação que incluem: fadiga, náuseas, vômito, dor, dispnéia, insônia, perda de apetite, constipação, diarréia e dificuldades financeiras ${ }^{(11)}$.

Os itens de 1 a 28 do questionário são colocados em Escala do tipo Likert de quatro pontos, onde as respostas seguem o seguinte padrão: não, pouco, moderadamente e muito. E os itens 29 e 30 são apresentados em Escala do tipo Likert de sete pontos, onde um corresponde a péssimo e sete corresponde a ótimo.

Os dados foram organizados em planilha do Excel e analisados por meio de análise estatística descritiva das variáveis: idade, escolaridade, estado civil, esquema quimioterápico, náusea e vômito para cada ciclo.

Para a análise do instrumento EORTC QLQ-C30, foi estabelecido um escore bruto (EB) para cada escala, formado pela somatória do valor das alternativas apontadas em cada questão/item que compõem a escala e dividido pelo número de respostas.

\section{RESULTADOS}

Participaram do estudo 79 mulheres com idade entre 29 a 69 anos e idade média de 48,4 anos, o que demonstra tratar-se de uma amostra composta em sua maioria por mulheres jovens, representadas por $55,8 \%$ com idade de 29 a 50 anos. A maioria $(79,7 \%)$ delas era da raça branca; $60,7 \%$ eram casadas e quanto à escolaridade, 40,5\% tinham ensino fundamental incompleto (Tabela 1).

Em relação ao protocolo de tratamento utilizado, 48 mulheres receberam EC-T (epirrubicina, ciclofosfamida e docetaxel) ou EC-TH (epirrubicina, ciclofosfamida, docetaxel e trastuzumab) e 31 utilizaram FEC (fluouracil, epirrubicina e ciclofosfamida), tanto na neoadjuvancia quanto na adjuvância.

Das 79 mulheres que participaram do estudo, 93\% apresentaram náuseas e $87 \%$ vômitos, pelo menos uma vez durante o tratamento.

Dentre as mulheres que receberam EC-T/EC-TH, observaram-se 231 ocorrências de náuseas, 118 de vômitos, totalizando 349 eventos durante todos os ciclos. Na Tabela 2 estão apresentados os episódios de náuseas e vômitos de acordo com o ciclo de tratamento. As náuseas foram mais frequentes até o quarto ciclo, enquanto que a ocorrência de vômitos oscila durante o tratamento, entretanto foi mais predominante nos cinco primeiros. Importante ressaltar que a mesma mulher pode ter apresentado estes eventos adversos mais de uma vez durante o tratamento.

Para as mulheres que receberam o esquema FEC, observaram-se 131 eventos de náuseas, 94 de vômitos, totalizando 225 eventos. No decorrer do tratamento os dois eventos apresentaram pouca oscilação (Tabela 3).

$\mathrm{Na}$ Tabela 4 consta o desvio-padrão para os itens QV e náuseas e vômitos do EORTC QLQ C30 para os três momentos em que este questionário foi aplicado. Pode-se observar que, durante o tratamento, nas três aplicações do questionário, as médias das escalas de "Medida global de saúde", para as mulheres que utilizaram FEC foram 71,24; 76,88 e 73,39 respectivamente, enquanto que para as que utilizaram EC-T/EC-TH foram 77,08; 75,69 e 71,10 . Estes valores não apresentam muita variação durante o tratamento, fato que indica $\mathrm{QV}$ 
Tabela 1 - Distribuição das mulheres submetidas à quimioterapia para câncer de mama, segundo idade, estado civil e escolaridade (N=79). Ribeirão Preto, SP, 2010.

\begin{tabular}{lcc}
\hline \multicolumn{1}{c}{ Variáveis } & Número & $\%$ \\
\hline Idade (anos) & 18 & 22,8 \\
29 a 40 & 26 & 33,0 \\
41 a 50 & 27 & 34,1 \\
51 a 60 & 08 & 10,1 \\
61 a 69 & & \\
Estado Civil & 48 & 60,7 \\
Casada & 12 & 15,1 \\
Solteira & 11 & 13,9 \\
Separada & 08 & 10,1 \\
Viúva & & \\
Escolaridade & 32 & 40,5 \\
Fundamental incompleto & 11 & 13,9 \\
Fundamental completo & 11 & 13,9 \\
Médio incompleto & 10 & 12,6 \\
Médio completo & 03 & 3,8 \\
Superior incompleto & 10 & 12,6 \\
Superior completo & 02 & 2,5 \\
$\quad$ Analfabeta & & \\
\hline
\end{tabular}

Tabela 2 - Distribuição das mulheres submetidas à quimioterapia para câncer de mama, segundo náuseas e vômitos entre os ciclos, para os protocolos EC-T/EC-TH. Ribeirão Preto, SP, 2010.

\begin{tabular}{|c|c|c|c|c|c|c|c|c|c|}
\hline $\begin{array}{l}\text { Evento } \\
\text { Adverso }\end{array}$ & $\begin{array}{c}\text { Após } \\
\text { Ciclo } 1 \\
(\mathrm{~N}=48)\end{array}$ & $\begin{array}{c}\text { Após } \\
\text { Ciclo } 2 \\
(\mathrm{~N}=48)\end{array}$ & $\begin{array}{c}\text { Após } \\
\text { Ciclo } 3 \\
(\mathrm{~N}=48)\end{array}$ & $\begin{array}{c}\text { Após } \\
\text { Ciclo } 4 \\
(\mathrm{~N}=48)\end{array}$ & $\begin{array}{c}\text { Após } \\
\text { Ciclo } 5 \\
(\mathrm{~N}=47)\end{array}$ & $\begin{array}{c}\text { Após } \\
\text { Ciclo } 6 \\
(\mathrm{~N}=45)\end{array}$ & $\begin{array}{c}\text { Após } \\
\text { Ciclo } 7 \\
(\mathrm{~N}=41)\end{array}$ & $\begin{array}{c}\text { Após } \\
\text { Ciclo } 8 \\
(\mathrm{~N}=43)\end{array}$ & $\begin{array}{l}\text { ero } \\
\text { al }\end{array}$ \\
\hline & $32(66 \%)$ & $35(73 \%)$ & $35(73 \%)$ & $34(71 \%)$ & $28(59 \%)$ & 251 & $19(46 \%)$ & $23(5$ & \\
\hline ôm & $19(39 \%)$ & $15(31 \%)$ & $15(31 \%)$ & $18(37 \%)$ & $17(36 \%)$ & $12(27 \%)$ & $08(19 \%)$ & $14(32 \%)$ & 118 \\
\hline
\end{tabular}

estável durante o mesmo, independente das drogas utilizadas (Tabela 4).

Independente do protocolo utilizado houve pouca variação na média e no desvio-padrão dos domínios "Qualidade de vida global" e "náuseas e vômitos" em relação aos esquemas quimioterápicos (Tabela 4).

$\mathrm{Na}$ Tabela 5 está listada a variável "náusea e vômito" que apresentou diferença estatisticamente significante para as mulheres estudadas, de acordo com o protocolo quimioterápico utilizado. O coeficiente alfa de Cronbach para cada aplicação dos questionários foi de 0,890492 para antes do início do tratamento, 0,936392 para o questionário na metade e de 0,937639 para o final. Esses valores mostram uma alta consistência interna da escala e a similaridade entre os coeficientes mostra sua confiabilidade nessas variáveis.

\section{DISCUSSÃO}

As características sócio-demográficas como baixa escolaridade, idade acima de 40 anos e estado civil casada encontradas neste estudo corroboram com os achados de outro estudo nacional ${ }^{(12)}$. Carac- 
Tabela 3 - Distribuição das mulheres submetidas à quimioterapia para câncer de mama, segundo náuseas e vômitos entre os ciclos, para o protocolo FEC. Ribeirão Preto, SP, 2010.

\begin{tabular}{|c|c|c|c|c|c|c|c|}
\hline $\begin{array}{c}\text { Evento } \\
\text { Adverso }\end{array}$ & $\begin{array}{c}\text { Após } \\
\text { Ciclo } 1 \\
(\mathrm{~N}=31)\end{array}$ & $\begin{array}{c}\text { Após } \\
\text { Ciclo } 2 \\
(\mathrm{~N}=31)\end{array}$ & $\begin{array}{c}\text { Após } \\
\text { Ciclo } 3 \\
(\mathrm{~N}=31)\end{array}$ & $\begin{array}{c}\text { Após } \\
\text { Ciclo } 4 \\
(\mathrm{~N}=31)\end{array}$ & $\begin{array}{c}\text { Após } \\
\text { Ciclo } 5 \\
(\mathrm{~N}=31)\end{array}$ & $\begin{array}{c}\text { Após } \\
\text { Ciclo } 6 \\
(\mathrm{~N}=29)\end{array}$ & $\begin{array}{c}\text { Número } \\
\text { Total }\end{array}$ \\
\hline Náuseas & $22(71 \%)$ & $22(71 \%)$ & $20(64 \%)$ & $22(71 \%)$ & $23(74 \%)$ & $22(76 \%)$ & 131 \\
\hline Vômitos & $19(61 \%)$ & $11(35 \%)$ & $17(55 \%)$ & $15(48 \%)$ & $15(48 \%)$ & $17(59 \%)$ & 94 \\
\hline
\end{tabular}

Tabela 4 - Representação dos domínios “Qualidade de vida global” e "náuseas e vômitos” do EORTC QLQ C30 nos três momentos da avaliação das mulheres com câncer de mama (N=79). Ribeirão Preto, SP, 2010.

\begin{tabular}{|c|c|c|c|c|c|c|}
\hline Momento & Esquema & Variáveis & Média & Desvio-Padrão & Mínimo & Máximo \\
\hline \multirow{4}{*}{1} & \multirow{2}{*}{ ECT/ECTH } & ql & 77,08 & 23,91 & 0,00 & 100,00 \\
\hline & & nv & 10,42 & 18,07 & 0,00 & 66,67 \\
\hline & \multirow{2}{*}{ FEC } & ql & 71,24 & 27,54 & 0,00 & 100,00 \\
\hline & & nv & 4,84 & 10,71 & 0,00 & 50,00 \\
\hline \multirow{4}{*}{2} & \multirow{2}{*}{ ЕСТ/ЕCTH } & $q 1$ & 75,69 & 24,48 & 16,67 & 100,00 \\
\hline & & nv & 19,79 & 26,55 & 0,00 & 100,00 \\
\hline & \multirow{2}{*}{ FEC } & ql & 76,88 & 19,21 & 41,67 & 100,00 \\
\hline & & nv & 23,12 & 30,33 & 0,00 & 100,00 \\
\hline \multirow{4}{*}{3} & \multirow{2}{*}{ ECT/ECTH } & $q 1$ & 71,10 & 20,03 & 33,33 & 100,00 \\
\hline & & nv & 19,50 & 26,99 & 0,00 & 100,00 \\
\hline & \multirow{2}{*}{ FEC } & ql & 73,39 & 22,40 & 0,00 & 100,00 \\
\hline & & nv & 17,74 & 20,61 & 0,00 & 66,67 \\
\hline
\end{tabular}

Legenda: $\mathrm{ql}$ = Qualidade de vida global; nv = Náusea e Vômito

Tabela 5 - Comparações durante o tratamento da variável náusea e vômito do EORTC QLQ-C30 para as mulheres submetidas à quimioterapia para câncer de mama (N=79). Ribeirão Preto, SP, 2010.

\begin{tabular}{lccc}
\hline \multicolumn{1}{c}{ Comparações } & Diferenças & P-valor & IC (95\%) \\
\hline Variável Náusea e Vômito & & & \\
(Inicio - Meio) ECT/ECTH & $-9,3746$ & 0,0118 & $(-16,6440 ;-2,1052)$ \\
(Inicio - Fim) ECT/ECTH & $-9,1052$ & 0,0151 & $(-16,4239 ;-1,7864)$ \\
(Inicio - Meio) FEC & $-18,2789$ & 0,0001 & $(-27,3245 ;-9,2333)$ \\
(Inicio - Fim) FEC & $-12,9026$ & 0,0055 & $(-21,9483 ;-3,8570)$ \\
\hline
\end{tabular}

terísticas que requerem do profissional de saúde maior ênfase nos processos educativos, adequando a linguagem do processo ensino aprendizagem ao nível de entendimento das pacientes.

A ocorrência de $93 \%$ de náusea e $87 \%$ de vômito encontrado é superior a dos demonstrados na literatura. Em estudo que avaliou 35 mulheres com câncer de mama em quimioterapia adjuvante, os autores constataram que $57,1 \%$ das participantes relataram sintomas gastrintestinais como náuseas, vômitos, constipação e/ou diarréia ${ }^{(12)}$. Já os achados de outros estudos que também avaliaram mulheres 
com câncer de mama durante a quimioterapia com regimes de alto e moderado potencial emético, evidenciaram ocorrência de náusea em torno de 31 a $70 \%$ e de vômitos 13 a $45 \%{ }^{(8-9)}$.

As drogas utilizadas pelas participantes, em especial a ciclofosfamida e a epirrubicina, presentes em ambos os protocolos quimioterápicos apresentam potencial emético que varia de muito alto a alto, a depender da dose utilizada. Tais afirmações podem ser confirmadas ao analisar um estudo ${ }^{(14)} \mathrm{em}$ que praticamente todos os participantes que receberam doxorrubicina e ciclofosfamida apresentaram elevada incidência de náuseas.

Em avaliação de 75 mulheres com câncer de mama em tratamento quimioterápico e 75 em tratamento radioterápico, com aplicação do EORTC QLQ C30, estudiosos constataram que o grupo do tratamento quimioterápico apresentou diminuição da QV, na função física, no desempenho de papéis, na função cognitiva, de imagem corporal, função social e ansiedade entre as entrevistas antes do início do tratamento e ao final do mesmo. Relatam ainda que, neste mesmo período, as mulheres apresentaram aumento significativo da náusea, vômito, constipação e dos eventos adversos sistêmicos da terapia utilizada ${ }^{(14)}$.

Os resultados de um estudo prospectivo envolvendo um grupo de 200 pacientes encontraram que a associação dos sintomas, perda de apetite, náuseas e vômitos, teve um impacto negativo na QV, mais do que náusea, vômito e perda de apetite individualmente ${ }^{(8)}$.

No presente estudo o domínio "náusea e vômito” do EORTC QLQ C30 apresentou diferença estatisticamente significante para as mulheres estudadas. Dados que corroboram com resultados de estudo ${ }^{(9)}$ no qual $72 \%$ dos pacientes que sentiram náuseas declararam que esta toxicidade teve um efeito negativo na sua vida diária. Esse percentual aumentou para mais de $89 \%$, quando a intensidade de náusea foi significativa.

Apesar de os resultados deste estudo apontar para uma QV que se mantêm durante o tratamento quimioterápico, a maioria dos estudos evidencia interferência significativamente ruim da quimioterapia na QV das mulheres com câncer de mama ${ }^{(8-10,12-13,15)}$.

Este fato demonstra a importância de avaliações da mulher durante todo o tratamento em todos os seus aspectos, não se limitando apenas a avaliações e questionamentos acerca dos sinais e sintomas físicos, mas englobando também os aspectos psicossociais.

Neste processo contínuo e dinâmico, o papel dos enfermeiros é crucial, uma vez que são os profissionais que passam a maior parte do tempo, com o paciente. Por esta razão, eles podem estabelecer um diálogo, adquirir a confiança do paciente e podem esclarecer o conceito que a mulher tem de si mesma, fornecendo ajuda e bom senso, apontando o melhor caminho a seguir(16).

\section{CONCLUSÃO}

Independente das facilidades ou dificuldades do uso de instrumentos para avaliação de sinais e sintomas relacionados ao tratamento quimioterápico, na prática clínica é importante que os profissionais de saúde valorizem estes sintomas e estabeleçam cuidados individualizados aos usuários.

A disponibilidade de informações sobre o tratamento e orientações quanto ao manejo dos seus eventos adversos é crucial para o gerenciamento adequado das toxicidades gastrintestinais. $\mathrm{Na}$ impossibilidade ou inviabilidade do uso de instrumentos para esta avaliação, as queixas das pacientes devem ser valorizadas e um plano assistencial estabelecido de modo a suprir tais lacunas.

\section{REFERÊNCIAS}

1 Instituto Nacional de Câncer (INCA). Ações de enfermagem para o controle do câncer: uma proposta de integração ensino-serviço. $3^{\text {a }}$ ed. rev. atual. ampl. Rio de Janeiro: INCA; 2008.

2 Montazeri A. Health-related quality of life in breast cancer patients: a bibliographic review of the literature from 1974 to 2007. J Exp Clin Cancer Res. 2008;27(1):1-31.

3 Hesketh PJ. Drug therapy: chemotherapy-induced nausea and vomiting. N Engl J Med. 2008;358:2482-94.

4 Lotti RCB, Barra AA, Dias RC, Makluf ASD. Impacto do tratamento de câncer de mama na qualidade de vida. Rev Bras Cancerol. 2008;54(4):367-71.

5 National Cancer Institute. Common Terminology Criteria for Adverse Events (CTCAE) v.4.o [Internet]. 2009 [cited 2010 Jun 10]. Available from: http://evs.nci.nih.gov/ftp1/CTCAE/CTCAE_4.03_ QuickReference_5x7.pdf. 
6 Molassiotis A, Saunders MP, Valle J, Lorigan P, Wardley A, Levine E, et al. A prospective observational study of chemotherapy-related nausea and vomiting in routine practice in a UK cancer center. Support Care Cancer. 2008;16(2):201-8.

7 Jakobsen JN, Herrstedt J. Prevention of chemotherapy-induced nausea and vomiting in elderly cancer patients. Crit Rev Oncol Hematol. 2009;71:214-21.

8 Pirri C, Bayliss E, Trotter J, Olver IN, Katris P, Drummond P, et al. Nausea still the poor relation in antiemetic therapy? The impact on cancer patients' quality of life and psychological adjustment of nausea, vomiting and appetite loss, individually and concurrently as part of a symptom cluster. Support Care Cancer. 2012; 21(3):735-48.

9 Fernández-Ortega PP, Caloto MT, Chirveches EE, Marquilles RR, Francisco JS, Quesad AA . Chemotherapy - induced nausea and vomiting in clinical pratice: impacto on patients' quality of life. Support Care Cancer. 2012;20(12):3141-48.

10 Gridelli C, Haiderali AM, Russo MW, Blackburn LM, Lykopoulos K. Casopitant improves the quality of life in patients receiving highly emetogenic chemotherapy. Support Care Cancer. 2010;18(11):1437-1444.

11 Brabo EP, Paschoal ME, Biasoli I, Nogueira FE, Gomes MC, Gomes IP, et al. Brazilian version of the QLQ-LC13 lung cancer module of the European Organization for Research and Treatment of Cancer: preliminary reliability and validity report. Qual Life Res. 2006;15(9):1519-1524.

12 Nicolussi AC, Sawada NO. Qualidade de vida de pacientes com câncer de mama em terapia adjuvante. Rev Gaúcha Enferm.2011;32(4):759-66.

13 Roscoe JA, Morrow GR, Colagiuri B, Heckler CE, Pudlo BD, Colman L, et al. Insight in the prediction of chemotherapy-induced nausea. Support Care Cancer. 2010;18(7):869-76.

14 Browall M, Ahlberg K, Karlsson P, Danielson E, Persson LO, Gaston-Johansson F. Health-related quality of life during adjuvant treatment for breast cancer among postmenopausal women. Eur J Oncol Nurs. 2008;12(3):180-89.

15 Farrel C, Brearleyet SG, Pilling M, Molassiotis A. The impact of chemotherapy-related nausea on patients' nutritional status, psychological distress and quality of life. Support Care Cancer. 2012;2 1(1):59-66.

16 Alves PC, Santos MCL, Fernandes AFC. Stress and coping strategies for women diagnosed with breast cancer: a transversal study. Online Braz J Nurs [Internet]. 2012 [citado 2012 Dez 20];11(2):305-18. Disponível em: http://www.objnursing.uff.br/index. php/nursing/article/view/3714/html

\author{
Endereço do autor / Dirección del autor / \\ Author's address \\ Thais de Oliveira Gozzo \\ Escola de Enfermagem de Ribeirão Preto, USP \\ Av. Bandeirantes, 3900, Monte Alegre \\ 14040-902, Ribeirão Preto, São Paulo \\ E-mail: thaisog@eerp.usp.br
}

Recebido em: 12.03.2013

Aprovado em: 21.08.2013 